\title{
Nursing of an Infant Diagnosed with Neonatal Intrahepatic Cholestasis Caused by Citrin Deficiency (NICCD) with Respiratory Syncytial Virus (RSV) Infection: A Case Report
}

\author{
Lingli Cai ${ }^{1}$, Yuanzong Song ${ }^{2}$, Ning Zhang ${ }^{1}$, Meixue Chen ${ }^{1}$, Weiju Chen ${ }^{1}$, Qingran Lin ${ }^{3,}$ * \\ ${ }^{1}$ School of Nursing, Jinan University, Guangzhou, China \\ ${ }^{2}$ Department of Pediatrics, The First Affiliated Hospital of Jinan University, Guangzhou, China \\ ${ }^{3}$ Department of Nursing, The First Affiliated Hospital of Jinan University, Guangzhou, China
}

Email address:

qingranlin@126.com (Qingran Lin), cailingli245@163.com (Lingli Cai)

${ }^{*}$ Corresponding author

\section{To cite this article:}

Lingli Cai, Yuanzong Song, Ning Zhang, Meixue Chen, Weiju Chen, Qingran Lin. Nursing of an Infant Diagnosed with Neonatal Intrahepatic Cholestasis Caused by Citrin Deficiency (NICCD) with Respiratory Syncytial Virus (RSV) Infection: A Case Report. Clinical Medicine Research. Vol. 10, No. 1, 2021, pp. 31-34. doi: 10.11648/j.cmr.20211001.16

Received: February 5, 2021; Accepted: February 15, 2021; Published: February 26, 2021

\begin{abstract}
Background: Citrin deficiency is an autosomal recessive genetic disorder caused by SLC25A13 genetic mutations. Understandably, Neonatal Intrahepatic Cholestasis caused by Citrin Deficiency (NICCD) was one of its clinical phenotypes, usually occurred in the neonatal period or infancy. The effective treatment of dietary intervention and symptomatic support may provide an avenue for clinicians to reduce liver damage and improve clinical prognosis. Objective: We report a 5-month-old infant diagnosed with Neonatal Intrahepatic Cholestasis caused by Citrin Deficiency (NICCD), coexisting Respiratory Syncytial Virus (RSV) infection. Method: The patient presented with rhinorrhea and cough, jaundiced skin and sclera, fat faces and hepatomegaly. Simultaneously, the child's mother performed obvious anxious emotion. Infection control, nutrition management, and psychological guidance for the caregiver during hospitalization to discharge were achieved. Meanwhile, effective health education also plays a good role in these interventions. Result: After half a month, the patient's infection was given controlled. Jaundice and hepatomegaly were improved gradually. Followeing up for 2 months, the clinical symptoms for NICCD were disappeared and the caregiver's anxiety was alleviated effectively. The importance of follow-up and support longitudinally to improve the quality of life for patients is emphasized. Conclusion: Therefore, this study provided primary while valuable nursing care experience for NICCD with RSV infection patients.
\end{abstract}

Keywords: Citrin Deficiency, SLC25A13, Nursing, Infant, Case Report

\section{Introduction}

Citrin Deficiency $(\mathrm{CD})$ is an autosomal recessive disease caused by SLC25A13 genetic mutations which causes three age-dependent clinical phenotypes, namely, neonatal intrahepatic cholestasis caused by citrin deficiency (NICCD) in infants, failure to thrive and dyslipidemia caused by citrin deficiency (FTTDCD) in older children, and adult-onset type II citrullinemia (CTLN2) in adults [1]. CD was first discovered in Japan [2], and the estimated frequency of patients is $1 / 7100$ in the Japanese population [3]. Up to now, it has been thought to a worldwide pan-ethnic disease [4]. Two SLC25A13 pathogenic allele mutations are recognized as CD
[5]. The clinical manifestation and laboratory inspection of NICCD are complex, they usually present with cholestatic jaundice, decreased alanine aminotransferase (ALT) to aspartate acid transaminase (AST) ratio, hypoglycemia, high levels of alpha-fetoprotein (AFP), and fatty liver, comfortingly, with symptoms usually self-resolving by the first years $[1,6]$. In this case report, we summarize the nursing experience and introduce a guideline for the effective management of NICCD with RSV infection patients. 


\section{Case Presentation}

A 5-month Chinese boy presented with a 3-month history of jaundice. The full-term infant weighed $2.7 \mathrm{~kg}$ and measured $48 \mathrm{~cm}$ at birth. The Apgar score was 10 . About three months after birth, his parent found jaundiced skin and sclera but did not pay much attention. On October 7, 2020, the infant underwent protect liver medicine in one hospital, but his liver function was still abnormal. In order to find the etiology, on October 18, 2020, the 5-month infant was accepted by the pediatric department of our hospital due to "jaundiced sclera, a cough and runny nose". At admission, vital signs remained stable, exhibiting normal development. The jaundiced skin and sclera were slight, and the faces were fat. The respiration of both lungs is thick and headed with scattered soft moist rales and sputum purrs. The liver was palpable $3.5 \mathrm{~cm}$ below the right subcostal margin with medium texture, and the speen was negative. Blood routine examination revealed white blood count (WBC) $14.64 \times 109 / \mathrm{L}$, neutrophil 19.3\%, lymphocyte
$73.9 \%$, monocyte 6.40\%, hemoglobin (HGB) 118/L, C-reaction protein (CTR) $2.5 \mathrm{mg} / \mathrm{L}$. Chest DR showed double lung texture slightly increased, thickened. Blood biochemical examination was abnormal. (Table 1) The serum zinc level was desperately insufficient at $4.8 \mathrm{umol} / \mathrm{L}$ and AFP was greatly elevated to $78307.1 \mathrm{ng} / \mathrm{ml}$. Plasmo amino acid analysis showed citrulline $62.9 \mu \mathrm{mol} / \mathrm{L}$ (reference ranges 7.00-35.00 $\mu \mathrm{mol} / \mathrm{L}$ ), methionine $299.0 \mu \mathrm{mol} / \mathrm{L}$ (reference ranges $5.00-34.00 \mu \mathrm{mol} / \mathrm{L}$ ) and lysine $199.3 \mu \mathrm{mol} / \mathrm{L}$ (reference ranges 52.00-183.00 $\mu \mathrm{mol} / \mathrm{L}$ ), elevating significantly. Citrin deficiency was suspected. And then the genotype shows c.328+6A>G/c.851_854delGTAT. He demonstrably was diagnosed with NICCD at his age of 5 months and 22 days.

Written informed consent was obtained from the patient's parents. All procedures performed in studies involving human participants were in accordance with the ethical standards of the institutional and/or national research committee(s) and with the Helsinki Declaration (as revised in 2013).

Table 1. Laboratory results over time in the patient.

\begin{tabular}{|c|c|c|c|c|c|c|}
\hline \multirow{2}{*}{ Index (Reference Range) } & \multicolumn{6}{|l|}{ Time } \\
\hline & $20201007^{a}$ & 20201011 & $20201018^{b}$ & 20201029 & $20201104^{\mathrm{c}}$ & 20201229 \\
\hline ALT (9-50U/L) & 43 & 34 & 56 & 46 & 45 & 69 \\
\hline $\operatorname{AST}(15-40 \mathrm{U} / \mathrm{L})$ & 108 & 80 & 117 & 66 & 67 & 68 \\
\hline GGT (10-60U/L) & 343 & 281 & 452 & 504 & 385 & 122 \\
\hline $\operatorname{ALP}(40-500 \mathrm{U} / \mathrm{L})$ & 1459 & 615 & 699 & 643 & 590 & 378 \\
\hline TP $(65-85 \mathrm{~g} / 1)$ & 47.5 & 55.2 & 52.4 & 59.4 & 56.4 & 56.5 \\
\hline ALB $(40-55 \mathrm{~g} / 1)$ & 31.5 & 36.6 & 36.7 & 41.4 & 40.7 & 40.8 \\
\hline Tbil (5.1-23umol/1) & 133.4 & 53.3 & 29 & 20.5 & 18.9 & 5.5 \\
\hline Dbil (0.6-6.8umol/1) & 57.4 & 41.2 & 22.6 & 15.6 & 13.8 & 2.5 \\
\hline Ibil (1.7-17umol/1) & 76 & 12.1 & 6.4 & 4.9 & 5.1 & 3 \\
\hline TBA (0-10umol/1) & 311.69 & 143.6 & 180.5 & 175.5 & 147.4 & 7.9 \\
\hline $\operatorname{AFP}(0-9 \mathrm{ng} / \mathrm{ml})$ & $>80000$ & $>80000$ & 78307.1 & - & 8279.33 & 269.23 \\
\hline $\mathrm{Zn}(7.65-25.5 \mathrm{umol} / \mathrm{L})$ & - & - & 4.8 & - & 6.4 & 10.8 \\
\hline $25-(\mathrm{OH})-\mathrm{Vit}(\geq 20 \mathrm{ng} / \mathrm{ml})$ & - & - & 17.1 & - & 30 & 58.2 \\
\hline
\end{tabular}

${ }^{\mathrm{a}}$ another hospital; ${ }^{\mathrm{b}}$ our hospital pediatrics department; ${ }^{\mathrm{c}}$ pediatric outpatient. ALT, alanine aminotransferase; AST, aspartate aminotransferase; GGT, $\gamma$-glutamyl transpeptidase; ALP, alkaline phosphatase; TP, total protein; Alb, albumin; Tbil, total bilirubin; Dbil, direct bilirubin; Ibil, indirect bilirubin; TBA, total bile acids; AFP, alpha-fetoprotein; Zn, Zinc; -, not tested.

The infant's inflammatory factors increased, his pulmonary function was damaged. After admission, intravenous Piperacillin $(50 \mathrm{mg} / \mathrm{kg})$ was initiated. Meanwhile, ambroxol hydrochloride intravenous drip combined with ventolin and budesonide aerosol inhalation were given for relieving cough phlegm. He was breastfeeded after birth, and changed to a lactose-free and medium-chain triglyceride (MCT)-enriched formula on October 11, 2020. Given the clinical features consistent with NICCD, the special formula was administered. Consider the susceptibility to hypoglycemia, he was given about $90 \mathrm{ml} / 2-3 \mathrm{~h}$ to supplement enough energy. The fat-soluble vitamins were also given. On the second day after admission, the child appeared anhelation and wheezing, intravenous methylprednisolone was given for 3 days and ribavirin was added to aerosol inhalation as adjusted therapies for resisting infection. The infant choked suddenly when drinking milk in the morning. Stop feeding and keeping lateral decubitus urgently. After the little boy's breathing pattern returning to normal, the responsible nurse coach his mother to adjust the angle of the bottle to slow down the feeding speed. The responsible nurse needs to identify hypoglycemia and disturbance in respiration when walks around the hospital every day and night. After 11 days of continuous treatment and nursing, the patient's jaundice and liver function got improved gradually. His pulmonary infection was effectively controlled, then discharged in a stable condition. Health education was advised to improve caregiver's home nursing skills. Depending on the disease conditions of the infant and the nutritional care guidelines [7-8], doctors and responsible nurses assist the family members to make personalized dietary plans. A kind of low-calorie, high-protein, and high-fat dietary pattern is required to adhere. Eggs, fish, meat, beans, and seafood are suitable. At the age of 6 months and 7 days, the infant received the first bite of side food- a spoonful of egg yolk. He had an interest in food and kept a good appetite. Half a month later, he ate a quarter of an egg and a spoonful of cod and milk $150 \mathrm{ml}$ at lunch. At the age of 7 months and 17 days, his bilirubin indices returned to routine and hepatomegaly 
resolved gradually. The patient was $69.6 \mathrm{~cm}$ in height, $8.55 \mathrm{~kg}$ in weight, and $45 \mathrm{~cm}$ in head circumference, with optimal growth. Fortunately, he also received adequate and safe complementary foods.

However, as the primary caregiver, the mother had an unpleasant experience. Before the child had definitely been diagnosed with NICCD, she suffered from obvious self-blame even weight loss due to failing to take care of baby. Meanwhile, she worried about worsening of symptoms and delaying in treatment or inappropriate. Understandably, that's the main cause mother's stress and frustration. Then, questionnaires assessing anxiety and depression were completed. The score of the Self-Rating Anxiety Scale (SAS) was 57, indicating mild anxiety, and Self-Rating Depression Scale (SDS) scored 33, indicating normal. Hamilton Anxiety Scale (HAMA) scored 11, showing the possibility of anxiety. After admission, considering the parents' educational level, the charge nurse explained the illness and treatment measures intelligibly. Use well-intentioned communication, like being a good listener, by a thumb-up, giving a warm hug and so on. In addition, the charge nurse shared the baby's daily care skills, such as feeding pattern, daily activity, and care of childhood infection. On November 4, 2020, some caregivers of patients diagnosed with NICCD who are of different ages introduced to the mother, likely, a 14-year-old patient, a 1-year-old patient and a 6-month-old patient. Encourage sharing valuable experience unselfishly. During the outpatient follow-up, the liaison nurse asked the mother to "Teach-Back" information in order to supplement with caregiving skills. The mother's anxiety alleviated gradually on subsequent follow up in our clinic. On December 29, the SAS and HAMA decreased to 33 and 4 respectively, indicating normal. Due to two months of support and companionship, the caregiver's quality of life has also improved.

\section{Discussion}

NICCD is the most clinical phenotype of CD [9]. What recognized treatment effectively is "Lactose (Galactose)-Restricted, Medium-Chain Triglyceride (MCT) Supplemented Formula, high protein, low carbon hydration diet, supplement lipid-soluble vitamin and ursodeoxycholic acid "[10]. Most cases are a real good prognosis through dietary intervention and a few evolve into hypohepatia or even death [11]. After the dietary intervention, the case's jaundice improved rapidly and biochemical indexes gradually recovered. Actually, Respiratory Syncytial Virus (RSV) infection was susceptive at the beginning of October and lasted 33 weeks in China [12], focused on children aged 0-24 months [13], and was associated with significant morbidity and mortality [14]. Emphatically, infection is unamiable for NICCD [15]. And two cases reported by Zhang [16] and Chew [17] presented infection may trigger acute liver failure directly or indirectly. Here, the infant's liver function was influenced by RSV infection. Based on previous experience, we quickly brought the infection under control and avoided aggravating liver function lesion. Therefore, it is important for NICCD to do a good job of respiratory protection during the susceptible season.

Unfortunately, those parents of children with a chronic rare disease were more likely to mental imbalance, such as social isolation and vulnerability [18], who experienced inadequate supports and general misunderstandings from health care providers and social support workers [19]. Statistically, at least a third of parents suffered from depression and anxiety, whose children were with undiagnosed diseases, but they may be always overlooked by clinicians due to the ability to act on their child's behalf [20]. This case's caregiver exposed the psychological problems, especially anxiety when the child was with undiagnosed diseases. Ascertained anxious for what rapidly, and provided appropriate and requisite interventions, including emotion and problem-focused coping strategies. After providing effective interventions, the caregiver's anxiety has been significantly alleviated. It should be noted, therefore, that what the psychological status of caregivers, who brought up a baby diagnosed with a rare disease.

\section{Conclusion}

In conclusion, NICCD is a chronic rare disease, affecting the quality of life and mental health of the child and parents. There are few reports of the overall experience of nursing a child with NICCD, other than studies on pathogenesis and their pathophysiology. In this case report, we described the nursing experience of a five-month child diagnosed with NICCD and RSV infection. A good therapeutic effect was achieved by infection control, nutrition management, and psychological guidance for the caregiver during hospitalization to discharge. It also will be important for these patients to follow-up and support longitudinally and thereby improve the quality of life.

\section{Funding}

The present study was supported by Guangdong Education Department Foundation (No. 2016QTLXXM-37).

\section{Conflict of Interest}

All the authors do not have any possible conflicts of interest.

\section{Acknowledgements}

We thank the patient and family who attended this study. Thank all healthcare workers in the Department of Pediatric in the First Affiliated Hospital of Jinan University as well as the reviewers and editors.

\section{References}

[1] Saheki, T., \& Song, Y. Z. (2005). Citrin Deficiency. In M. P. Adam (Eds.) et. al., GeneReviews ${ }^{\circledR}$. University of Washington, Seattle. 
[2] Saheki, T., Nakano, K., Kobayashi, K., Imamura, Y., Itakura, Y., Sase, M., Hagihara, S., \& Matuo, S. (1985). Analysis of the enzyme abnormality in eight cases of neonatal and infantile citrullinaemia in Japan. Journal of inherited metabolic disease, $8(3), 155-156$

[3] Kikuchi, A., Arai-Ichinoi, N., Sakamoto, O., Matsubara, Y., Saheki, T., Kobayashi, K., Ohura, T., \& Kure, S. (2012). Simple and rapid genetic testing for citrin deficiency by screening 11 prevalent mutations in SLC25A13. Molecular genetics and metabolism, 105 (4), 553-558.

[4] Dimmock, D., Maranda, B., Dionisi-Vici, C., Wang, J., Kleppe, S., Fiermonte, G., Bai, R., Hainline, B., Hamosh, A., O'Brien, W. E., Scaglia, F., \& Wong, L. J. (2009). Citrin deficiency, a perplexing global disorder. Molecular genetics and metabolism, $96(1), 44-49$.

[5] National Health Commission of the People's Republic of China Notice of the General Office of the National Health Commission on the Issuance of Guidelines for the Diagnosis and Treatment of Rare Diseases (2019 edition). [EB/OL]. [2019-02-27].

http://www.nhc.gov.cn/yzygj/s7659/201902/61d06b4916c348 e0810ce1 fceb844333.shtml

[6] Saheki, T., Inoue, K., Tushima, A., Mutoh, K., \& Kobayashi, K. (2010). Citrin deficiency and current treatment concepts. Molecular genetics and metabolism, 100 Suppl 1, S59-S64.

[7] Raphael B. P. (2015). Nutritional management in cholestatic liver disease. World review of nutrition and dietetics, 113, 178-181.

[8] Young, S., Kwarta, E., Azzam, R., \& Sentongo, T. (2013). Nutrition assessment and support in children with end-stage liver disease. Nutrition in clinical practice: official publication of the American Society for Parenteral and Enteral Nutrition, 28 (3), 317-329.

[9] Xueyuan Gu. Clinical Geneticmetabolic Diseases. Beijing: People's Medical Publishing House. 2015: 84-89.

[10] Hayasaka, K., Numakura, C., Toyota, K., \& Kimura, T. (2012). Treatment with lactose (galactose)-restricted and medium-chain triglyceride-supplemented formula for neonatal intrahepatic cholestasis caused by citrin deficiency. JIMD reports, 2, 37-44.

[11] Sha Cai. Analysis of clinical characteristics of 709 cases with
Neonatal Intrahepatic cholestasis caused by Citrin deficiency [dissertation]. Guanzhou: Nanchang University, 2019. (in Chinese).

[12] Yu, J., Liu, C., Xiao, Y., Xiang, Z., Zhou, H., Chen, L., Shen, K., Xie, Z., Ren, L., \& Wang, J. (2019). Respiratory Syncytial Virus Seasonality, Beijing, China, 2007-2015. Emerging infectious diseases, 25 (6), 1127-1135.

[13] Toivonen, L., Karppinen, S., Schuez-Havupalo, L., Teros-Jaakkola, T., Mertsola, J., Waris, M., \& Peltola, V. (2020). Respiratory syncytial virus infections in children 0-24 months of age in the community. The Journal of infection, 80 (1), 69-75.

[14] Borchers, A. T., Chang, C., Gershwin, M. E., \& Gershwin, L. J. (2013). Respiratory syncytial virus--a comprehensive review. Clinical reviews in allergy \& immunology, 45 (3), 331-379.

[15] Abuduxikuer, K., Chen, R., Wang, Z. L., \& Wang, J. S. (2019). Risk factors associated with mortality in neonatal intrahepatic cholestasis caused by citrin deficiency (NICCD) and clinical implications. BMC pediatrics, 19 (1), 18.

[16] Zhang, M. H., Gong, J. Y., \& Wang, J. S. (2015). Citrin deficiency presenting as acute liver failure in an eight-month-old infant. World journal of gastroenterology, 21 (23), 7331-7334.

[17] Chew, H. B., Ngu, L. H., Zabedah, M. Y., Keng, W. T., Balasubramaniam, S., Hanifah, M. J., \& Kobayashi, K. (2010). Neonatal intrahepatic cholestasis associated with citrin deficiency (NICCD): a case series of 11 Malaysian patients. Journal of inherited metabolic disease, 33 Suppl 3, S489-S495.

[18] Pelentsov, L. J., Laws, T. A., \& Esterman, A. J. (2015). The supportive care needs of parents caring for a child with a rare disease: A scoping review. Disability and health journal, 8 (4), 475-491.

[19] Pelentsov, L. J., Fielder, A. L., \& Esterman, A. J. (2016). The Supportive Care Needs of Parents With a Child With a Rare Disease: A Qualitative Descriptive Study. Journal of pediatric nursing, 31 (3), e207-e218.

[20] McConkie-Rosell, A., Hooper, S. R., Pena, L., Schoch, K., Spillmann, R. C., Jiang, Y. H., Cope, H., Undiagnosed Diseases Network, Palmer, C., \& Shashi, V. (2018). Psychosocial Profiles of Parents of Children with Undiagnosed Diseases: Managing Well or Just Managing?. Journal of genetic counseling, 27 (4), 935-946. 